\title{
Оценка изотопного состава стандартных образцов урана методом полного испарения с помощью термоионизационного масс-спектрометра*
}

3. Палач

УДК 543.51

\begin{abstract}
Оценка изотопного состава топлива - важная задача для атомной энергетики. Известно несколько основных методик по определению изотопного состава тяжелых элементов, например урана, как более точных и длительных, так и экспрессных. Для производства с большим количеством образцов оптимален метод полного испарения, обеспечивающий высокую степень автоматизации и приемлемую точность анализа. Полное испарение также позволяет снижать уровень квалификации в подготовке образцов, уменьшая тем самым погрешности в результатах. В работе проведена проверка стандартных образцов состава NBS U010, NBL U030а и NBL U050 методом полного испарения с помощью термоионизационного масс-спектрометра Phoenix (Isotopx, Великобритания). Статистическая обработка результатов серии экспериментов показала, что по сравнению с другими метод полного испарения лучше соответствует по точности требованиям к определению изотопного отношения ${ }^{235} \mathrm{U} / 238 \mathrm{U}$ и согласуется с литературными данными.
\end{abstract}

Ключевые слова: уран, метод полного испарения, термоионизационная массспектрометрия, изотопное отношение

Measurement of the isotopic composition of the fuel is an important task for the nuclear industry. Several basic techniques are known for determining the isotopic composition of heavy elements, such as uranium, both more accurate and long-lasting, and express. For production with a large number of samples, the method of total evaporation is optimal, providing a high degree of automation and acceptable accuracy of analysis. Total evaporation also allows to reduce the qualification level in sample preparation, thereby reducing errors in the results. In this work, standard samples of the composition NBS U010, NBL U030a, and NBL U050 were tested by the method of total evaporation using a Phoenix thermal ionization mass spectrometer (Isotopx, UK). Statistical processing of the results of a series of experiments showed that, in comparison with others, the complete evaporation method better meets the accuracy requirements for determining the ${ }^{235} \mathrm{U} / 238 \mathrm{U}$ isotopic ratio and is consistent with published data.

Keywords: uranium, total evaporation method, thermal ionization mass spectrometry, isotope ratio

\footnotetext{
* Перевод статьи Isotopx Application Brief N10311. Palacz Z. Measurement of NBS U010, NBL U030a and NBL U050 uranium standards by total evaporation using the Phoenix TIMS. - https://www.isotopx.com/wp-content/uploads/2015/01/N10311-NBS-U010-NBL-U030a-and-NBLU050-by-total-evaporation.pdf

1 Isotopx Ltd, Мидлвич, Чешир, Великобритания, moscow@textronica.com.
} 


\section{Введение}

В ряде ведущих стран существенная доля производимой энергии вырабатывается на атомных электростанциях. В состав ядерного топлива входит изотоп урана-235, который в природном уране составляет $0,7 \%$, а остальные 99,3\% - это изотоп U-238. Поэтому для начала урановую руду обогащают, прессуют и спекают в таблетки, добавляя необходимые компоненты и получая ТВЭЛы, из которых собирают ТВС (тепловыделяющие сборки). Топливо, содержащее уран, подвергают всестороннему контролю, так как его изотопный состав определяет энергетическую способность и стоимость. Один из физико-химических методов анализа - термоионизационная массспектрометрия (ТИМС). В ТИМС используется термическая ионизация вещества: на ленту из тугоплавкого металла наносят образец и разогревают электрическим током. Образующиеся ионы в вакуумной камере ускоряются сильным электрическим полем, порядка 8 кB, и, проходя через поперечное магнитное поле в приборе, под действием силы Лоренца меняют свою траекторию и разделяются на различные пучки в зависимости от соотношения массы и заряда. Регистрируя раздельные пучки ионов, можно определить сигналы, соответствующие конкретным изотопам. А отношение интенсивностей сигналов одновременно регистрируемых ионных пучков дает информацию об изотопном составе вещества.

Несмотря на то, что уран не имеет стабильной пары изотопов, есть ряд внешних методов для коррекции эффекта фракционирования, которое происходит во время анализа в ТИМС.

Так в качестве "золотого стандарта" принят метод двойного изотопного разведения (double spike) с помощью добавки ${ }^{233} \mathrm{U}-236 \mathrm{U}$ для учета эффекта фракционирования. Хотя эта процедура и обеспечивает высочайшее качество результата, но требует высокой квалификации и весьма дорога.

Более простой подход для учета эффекта фракционирования заключается в применении

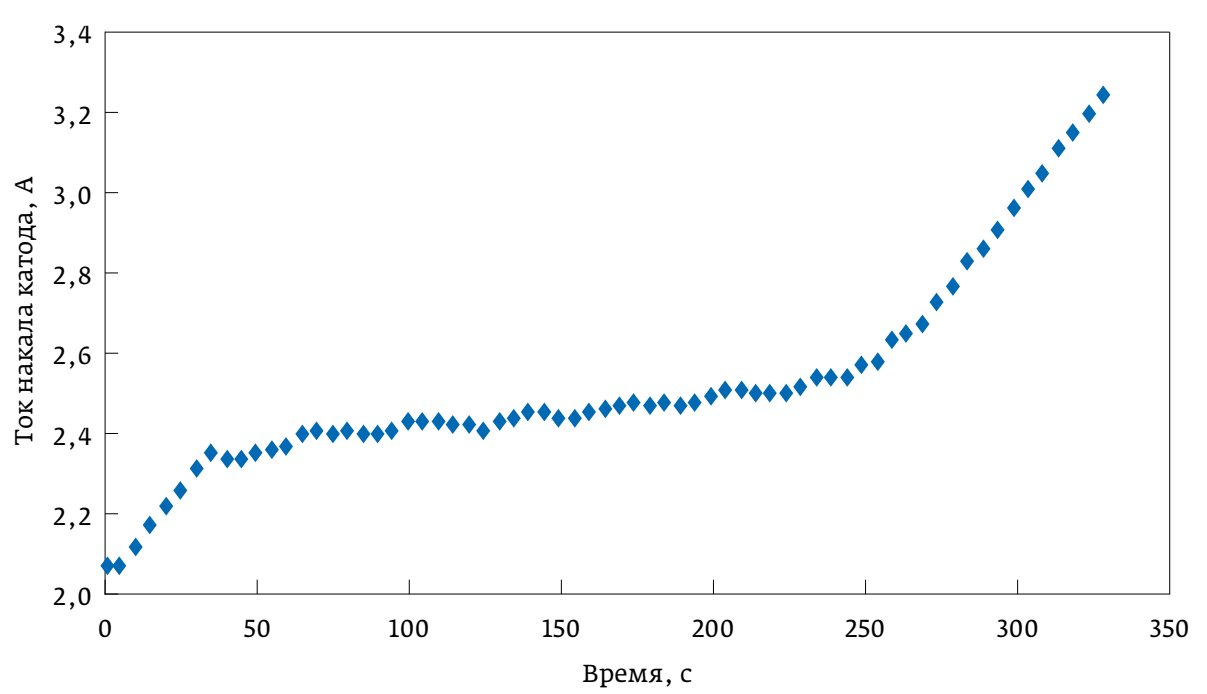

Puc. 2. Типичный профиль тока на катоде испарителя внешнего стандарта, измеренного совместно с образцами в пределах одной серии. При этом крайне важно использовать близкий по составу к образцам стандарт и обеспечить стабильность параметров анализа такой серии измерений. Эти факторы не позволяют полноценно автоматизировать процесс анализа, хоть и удается достичь точности $\sim 0,05 \%$ (RSD) для ${ }^{235} \mathrm{U} /{ }^{238} \mathrm{U}$.

Третий подход заключается в методе полного испарения образца (total evaporation). При этом поддерживается постоянной температура ионизирующего катода, в то время как температура на испаряющем катоде увеличивается, пока весь образец не испарится. Интегрирование ионных интенсивностей в течение всего измерения образца нивелирует эффект фракционирования и позволяет точно определить изотопные отношения. 


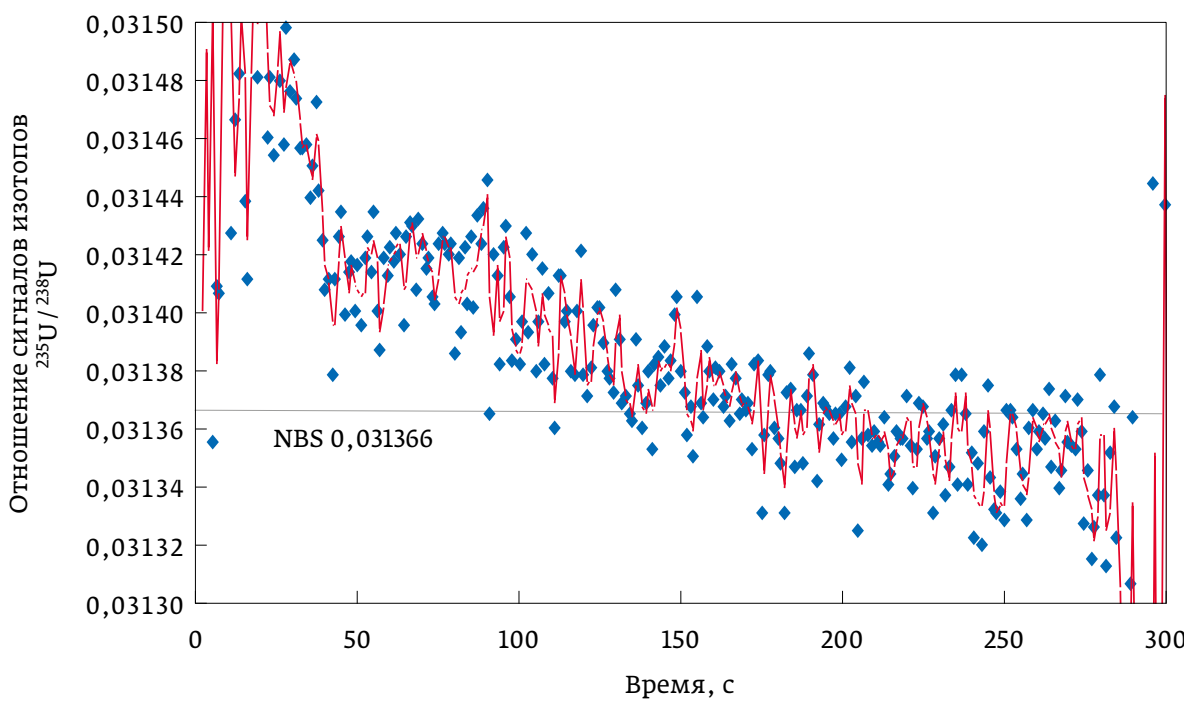

Puc. 3. Профиль отношения $235 \mathrm{U} / 238$ U для стандарта U030а при нанесении 200 нг

\section{Описание}

\section{эксперимента}

Измерения проводили на термоэмиссионном масс-спектрометре для сверхточного изотопного анализа Phoenix (Isotopx, Великобритания). Изотопическая чувствительность прибора по урану- $238 \pm 1$ а.е.м. менее 2 ppm, а при использовании дополнительных опций (WARP) снижается до $20 \mathrm{ppb}$.

Прибор оборудован высокочувствительными, малошумными, стабильными детекторами, которые могут измерять очень низкие ионные токи от нА вплоть до обнаружения одиночного иона $1,6 \cdot 10^{-19} \mathrm{~A}$.

Таблица 1. Обобщенные результаты измерений стандарта NBS U010, нагрузки 200 и 400 нг

\begin{tabular}{|c|c|c|c|c|c|c|c|}
\hline \multirow{2}{*}{$\begin{array}{l}200 \text { нг NBS } \\
\text { U010 }\end{array}$} & \multicolumn{4}{|c|}{ Суммарные интенсивности } & \multicolumn{3}{|c|}{ Отношения } \\
\hline & ${ }^{234} U_{\text {сумм }}, \Pi A$ & ${ }^{235} \mathrm{U}_{\text {сумм }}, \mathrm{HA}$ & ${ }^{236} U_{\text {сумм }}, \Pi \mathrm{A}$ & ${ }^{238} \mathrm{U}_{\text {сумм }}, \mathrm{HA}$ & ${ }^{234} \mathrm{U} /{ }^{238} \mathrm{U}_{\text {сумм }}$ & ${ }^{235} \mathrm{U} /{ }^{238} \mathrm{U}_{\text {сумм }}$ & ${ }^{236} \mathrm{U} /{ }^{238} \mathrm{U}_{\text {сумм }}$ \\
\hline & 0,57 & 0,10 & 0,74 & 9,9 & 0,000058 & 0,010140 & 0,000075 \\
\hline & 0,74 & 0,13 & 0,95 & 13 & 0,000056 & 0,010145 & 0,000073 \\
\hline & 0,62 & 0,11 & 0,79 & 11 & 0,000058 & 0,010145 & 0,000074 \\
\hline & 1,10 & 0,20 & 1,40 & 19 & 0,000055 & 0,010142 & 0,000072 \\
\hline & 1,40 & 0,27 & 1,80 & 26 & 0,000053 & 0,010149 & 0,000068 \\
\hline & 1,20 & 0,23 & 1,60 & 22 & 0,000056 & 0,010143 & 0,000073 \\
\hline & 2,00 & 0,35 & 2,50 & 35 & 0,000057 & 0,010146 & 0,000073 \\
\hline Среднее & 1,10 & 0,20 & 1,40 & 19 & 0,000056 & 0,010144 & 0,000072 \\
\hline SD & & & & & 0,000002 & 0,000003 & 0,000002 \\
\hline RSD & & & & & $3,0 \%$ & $0,03 \%$ & $2,9 \%$ \\
\hline \multirow{8}{*}{$\begin{array}{l}400 \text { нг NBS } \\
\text { U010 }\end{array}$} & \multicolumn{4}{|c|}{ Суммарные интенсивности } & \multicolumn{3}{|c|}{ Отношения } \\
\hline & ${ }^{234} U_{\text {сумм }}, \Pi \mathrm{A}$ & ${ }^{235} \mathrm{U}_{\text {сумм }}, \mathrm{HA}$ & ${ }^{236} U_{\text {сумм }}, \Pi A$ & ${ }^{238} \mathrm{U}_{\text {сумм }}, \mathrm{HA}$ & ${ }^{234} U /{ }^{238} U_{\text {сумм }}$ & ${ }^{235} \mathrm{U} /{ }^{238} \mathrm{U}_{\text {сумм }}$ & ${ }^{236} \mathrm{U} /{ }^{238} \mathrm{U}_{\text {сумм }}$ \\
\hline & 3,9 & 0,64 & 4,60 & 63,0 & 0,000062 & 0,010149 & 0,000073 \\
\hline & 1,6 & 0,30 & 2,10 & 29,0 & 0,000055 & 0,010146 & 0,000073 \\
\hline & 2,0 & 0,36 & 2,60 & 36,0 & 0,000056 & 0,010149 & 0,000074 \\
\hline & 1,0 & 0,19 & 1,40 & 19,0 & 0,000054 & 0,010151 & 0,000072 \\
\hline & 2,2 & 0,40 & 2,90 & 39,0 & 0,000057 & 0,010145 & 0,000073 \\
\hline & 1,5 & 0,28 & 1,90 & 28,0 & 0,000056 & 0,010147 & 0,000071 \\
\hline Среднее & 2,05 & 0,361 & 2,59 & 35,6 & 0,000057 & 0,010148 & 0,000073 \\
\hline SD & & & & & 0,000003 & 0,000002 & 0,000001 \\
\hline RSD & & & & & $4,9 \%$ & $0,02 \%$ & $1,4 \%$ \\
\hline
\end{tabular}

SD - Standard Deviation, среднеквадратическое (стандартное) отклонение.

RSD - Relative Standard Deviation, относительное стандартное отклонение. 
Для оценки суммарного испарения на Phoenix ТимС проведены эксперименты с нагрузками на катод: 200 и 400 нг стандарты NBS U010 и NBL U030a, а также 200 нг - NBL U050.

Перед началом испарения образцов был получен, сфокусирован, оптимизирован и отцентрирован ионный пучок $150 \mathrm{MB}$ с изотопом ${ }^{238} \mathrm{U}$. Базовую линию записывали в течение 60 с при $\pm 0,5$ а.е. м. Затем начался процесс испарения - при этом каждую секунду производилось интегрирование и увеличение тока испаряющего катода с шагом 40 мА до достижения сигнала 4 В для ${ }^{238} \mathrm{U}$. Далее сигнал удерживался на уровне 4 В за счет увеличения тока на катоде испарения. Типичный плоский профиль сигнала представлен на рис. 1.

Когда образец почти израсходован, ток катода испарителя снова увеличивался со скоростью $0,04 \mathrm{~A} / \mathrm{c}$ (рис. 2), стремясь поддерживать интенсивность сигнала на уровне 4 В. Резкое увеличение тока катода приводит к быстрому отжигу образца и потере сигнала. Сбор данных прекращается, когда сигнал падает до 150 мВ, а ток накала катода поднимается выше 3,3 А.
На рис. 3 показано, как меняется соотношение ${ }^{235} \mathrm{U} /{ }^{238} \mathrm{U}$ в течение процесса испарения. Начало и "Хвост" графика имеют большой разброс из-за низкой интенсивности сигнала, а центральная часть демонстрирует уменьшение отношения, пересекая линию, соответствующую сертифицированному значению ${ }^{235} \mathrm{U} /{ }^{238} \mathrm{U}$ для стандарта U030a.

\section{Результаты}

\section{Стандартный образец U010}

Результаты серии анализов нанесений 200 и 400 нг стандарта U010 приведены в табл. 1. Эти соотношения получены суммированием интенсивности по всем измерениям в ходе анализа.

Среднее значение ${ }^{235} \mathrm{U} / 238 \mathrm{U}$ для 200 нг U010 составило $0,010144 \pm 0,03 \%$ RSD, а для нагрузки $400 \mathrm{Hг-}$ $0,010148 \pm 0,02 \%$ RSD.

Среднее значение для двух нанесений $0,010146 \pm 0,03 \%$ RSD, а сертифицированное значение $0,01014 \pm 0,1 \%$, поэтому коэффициент остаточного смещения между ними 0,01014/ $0,010146=0,999413$.

Таблица 2. Обобщенные результаты измерений стандарта NBL U030а, нагрузки 200 и 400 нг

\begin{tabular}{|c|c|c|c|c|c|c|c|}
\hline \multirow{2}{*}{$\begin{array}{l}200 \text { нг NBL } \\
\text { U030a }\end{array}$} & \multicolumn{4}{|c|}{ Суммарные интенсивности } & \multicolumn{3}{|c|}{ Отношения } \\
\hline & ${ }^{234} U_{\text {сумм }}, \Pi A$ & ${ }^{235} U_{\text {сумм }}, \mathrm{HA}$ & ${ }^{236} U_{\text {сумм }}, \Pi A$ & ${ }^{238} \mathrm{U}_{\text {сумм }}, \mathrm{HA}$ & ${ }^{234} \mathrm{U} /{ }^{238} \mathrm{U}_{\text {сумм }}$ & ${ }^{235} \mathrm{U} /{ }^{238} \mathrm{U}_{\text {сумм }}$ & ${ }^{236} \mathrm{U} / 238 \mathrm{U}_{\text {сумм }}$ \\
\hline & 5,0 & 0,55 & 0,12 & 17,0 & 0,000287 & 0,031382 & 0,000007 \\
\hline & 7,4 & 0,81 & 0,24 & 26,0 & 0,000286 & 0,031371 & 0,000009 \\
\hline & 3,0 & 0,32 & 0,86 & 10,0 & 0,000289 & 0,031384 & 0,000008 \\
\hline & 3,2 & 0,35 & 0,12 & 11,0 & 0,000288 & 0,031388 & 0,000011 \\
\hline & 1,8 & 0,20 & 0,062 & 6,4 & 0,000286 & 0,031387 & 0,000010 \\
\hline & 1,8 & 0,20 & 0,068 & 6,3 & 0,000288 & 0,031390 & 0,000011 \\
\hline & 4,8 & 0,53 & 0,13 & 17,0 & 0,000288 & 0,031392 & 0,000008 \\
\hline Среднее & 3,9 & 0,42 & 0,12 & 13,0 & 0,000287 & 0,031385 & 0,000009 \\
\hline SD & & & & & 0,000001 & 0,000007 & 0,000001 \\
\hline RSD & & & & & $0,4 \%$ & $0,023 \%$ & $16 \%$ \\
\hline \multirow{6}{*}{$\begin{array}{l}400 \mathrm{Hг} \mathrm{NBL} \\
\text { U030a }\end{array}$} & \multicolumn{4}{|c|}{ Суммарные интенсивности } & \multicolumn{3}{|c|}{ Отношения } \\
\hline & ${ }^{234} U_{\text {сумм }}, \Pi A$ & ${ }^{235} \mathrm{U}_{\text {сумм }}, \mathrm{HA}$ & ${ }^{236} U_{\text {сумм }}, \Pi A$ & ${ }^{238} U_{\text {сумм }}, H A$ & ${ }^{234} \mathrm{U} /{ }^{238} \mathrm{U}_{\text {сумм }}$ & ${ }^{235} \mathrm{U} /{ }^{238} \mathrm{U}_{\text {сумм }}$ & ${ }^{236} \mathrm{U} /{ }^{238} \mathrm{U}_{\text {сумм }}$ \\
\hline & 8,1 & 0,88 & 0,25 & 28,0 & 0,000288 & 0,031388 & 0,000009 \\
\hline & 11,0 & 1,2 & 0,36 & 39,0 & 0,000289 & 0,031388 & 0,000009 \\
\hline & 4,4 & 0,47 & 0,17 & 15,0 & 0,000290 & 0,031392 & 0,000011 \\
\hline & 5,9 & 0,64 & 0,22 & 20,0 & 0,000291 & 0,031388 & 0,000011 \\
\hline Среднее & 7,4 & 0,81 & 0,25 & 26,0 & 0,000290 & 0,031389 & 0,000010 \\
\hline SD & & & & & 0,000001 & 0,000002 & 0,000001 \\
\hline RSD & & & & & $0,4 \%$ & $0,006 \%$ & $11,5 \%$ \\
\hline
\end{tabular}


Таблица 3. Обобщенные результаты измерений стандарта NBS U050, нагрузка 200 нг

\begin{tabular}{|c|c|c|c|c|c|c|c|}
\hline \multirow{2}{*}{$\begin{array}{l}200 \mathrm{Hг} \text { NBL } \\
\text { U050 }\end{array}$} & \multicolumn{4}{|c|}{ Суммарные интенсивности } & \multicolumn{3}{|c|}{ Отношения } \\
\hline & ${ }^{234} U_{\text {сумм }} \Pi A$ & ${ }^{235} U_{\text {сумм }}, \mathrm{HA}$ & ${ }^{236} U_{\text {сумм }}, \Pi \mathrm{A}$ & ${ }^{238} \mathrm{U}_{\text {сумм }}, \mathrm{HA}$ & ${ }^{234} U /{ }^{238} U_{\text {сумм }}$ & ${ }^{235} \mathrm{U} /{ }^{238} \mathrm{U}_{\text {сумм }}$ & ${ }^{236} \mathrm{U} /{ }^{238} \mathrm{U}_{\text {сумм }}$ \\
\hline & 3,8 & 0,67 & 6,4 & 13,0 & 0,000296 & 0,052767 & 0,000507 \\
\hline & 11,0 & 1,99 & 18,0 & 36,0 & 0,000295 & 0,052791 & 0,000509 \\
\hline & 5,4 & 0,98 & 9,4 & 18,0 & 0,000295 & 0,052776 & 0,000509 \\
\hline & 3,6 & 0,64 & 6,2 & 12,0 & 0,000296 & 0,052785 & 0,000511 \\
\hline & 5,8 & 1,0 & 10,0 & 19,0 & 0,000299 & 0,052792 & 0,000513 \\
\hline & 1,9 & 0,34 & 3,3 & 6,4 & 0,000295 & 0,052802 & 0,000513 \\
\hline & 7,7 & 1,4 & 13,0 & 26,0 & 0,000295 & 0,052789 & 0,000513 \\
\hline & 9,8 & 1,7 & 17,0 & 33,0 & 0,000296 & 0,052796 & 0,000511 \\
\hline Среднее & 6,1 & 1,1 & 10,0 & 21,0 & 0,000296 & 0,052787 & 0,000511 \\
\hline SD & & & & & 0,000001 & 0,000011 & 0,000002 \\
\hline RSD & & & & & $0,4 \%$ & $0,02 \%$ & $0,43 \%$ \\
\hline
\end{tabular}

\section{Стандартный образец U030a}

Результаты анализа после нанесений 200 и 400 нг стандарта U030а приведены в табл. 2.

Для 200 нг U030а получено значение ${ }^{235} \mathrm{U} /{ }^{238} \mathrm{U}$ $0,031385 \pm 0,023 \%$ (RSD), а для 400 нг - 0,031389 $\pm 0,006 \%$ (RSD). Усредненное значение для двух нанесений $0,031386 \pm 0,02 \%$ (RSD).

Скорректировав отношение для U030a на коэффициент, рассчитанный при измерении стандарта U010, получаем отношение 0,031368 , которое находится в пределах доверительного интервала сертифицированного значения - 0,031367. Скорректированное отношение также соответствует опубликованному в работе Рихтера и Голдберга*: $0,031369 \pm 0,009 \%$, которое определено методом полного испарения с нагрузками в нескольких мкг образца.

\section{Стандартный образец U050}

Восемь измерений с нагрузкой 200 нг U050 дают среднее значение ${ }^{235} \mathrm{U} /{ }^{238} \mathrm{U} 0,052787 \pm 0,02 \%$ (RSD) (табл. 3). Скорректировав измеренное отношение для U050 на коэффициент, рассчитанный при измерении стандарта U010, получаем отношение 0,052756 . Скорректированная величина существенно отличается от сертифицированной - 0,052784, но идентична опубликованному в работе Рихтера и Голдберга (2003) значению 0,052756 в большой выборке. Видимо,

* Richter S. Goldberg S. A. Improved techniques for high accuracy isotope ratio measurements of nuclear materials using thermal ionization mass spectrometry. International Journal of Mass Spectrometry. 2003. V. 229. P. 181-197. сертифицированное значение для стандартного образца U050 не корректно.

\section{Заключение}

В результате проведенных экспериментов можно сделать следующие выводы.

- С помощью метода полного испарения на образцах с нагрузками в несколько сотен нанограммов получены значения изотопного отношения ${ }^{235} \mathrm{U} /{ }^{238} \mathrm{U}$ с точностью 0,03\% (RSD), которая превышает аналогичную величину в методе коррекции с использованием внешнего стандарта.

- Зафиксировано небольшое отклонение 0,06\% для измеренного отношения ${ }^{235} \mathrm{U} /{ }^{238} \mathrm{U}$, или $0,02 \%$ на единицу массы, причем суммированные коэффициенты несколько превышают сертифицированное значение.

- Отклонение незначительно растет с увеличением выборки.

- Величина отклонения отражает закономерность между U010 и U030a, и поэтому результат измерений может быть скорректирован .

- Скорректированное отношение ${ }^{235} \mathrm{U} /{ }^{238} \mathrm{U}$ для U050 не попадает в доверительный интервал, определяемый погрешностью сертифицированного значения, но согласуется с величиной, полученной Рихтером и Голдбергом (2003) на другом типе ТИМС.

Перевод Григория Карабаева, ведущего специалиста группы компаний Textronica AG/ "МС-АНАЛИТИКА» 


\section{DiscovIR-LC}

DEPOSITION AND DETECTION SYSTI

SPECTRA

A N A L Y S I S

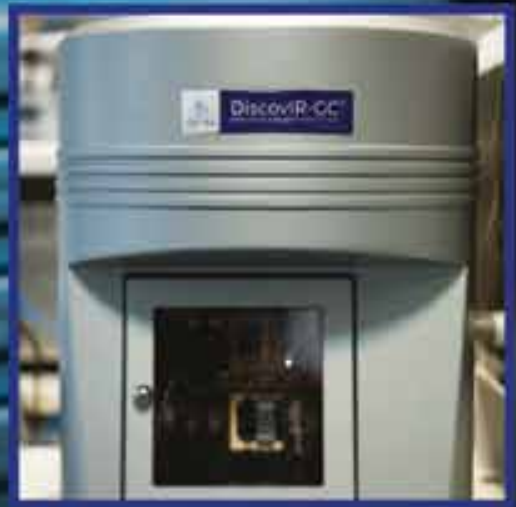

Системы ИК-Фурье-спектроскопии для газовой и жидкостной хроматографии

Системы DiscovIR от Spectra Analysis - это идеальное решение для идентификации компонентов в сложных смесях. Благодаря тому, что каждое вещество имеет свой уникальный ИК-спектр, с помощью систем DiscovIR можно быстро и легко проводить идентификацию соединений.

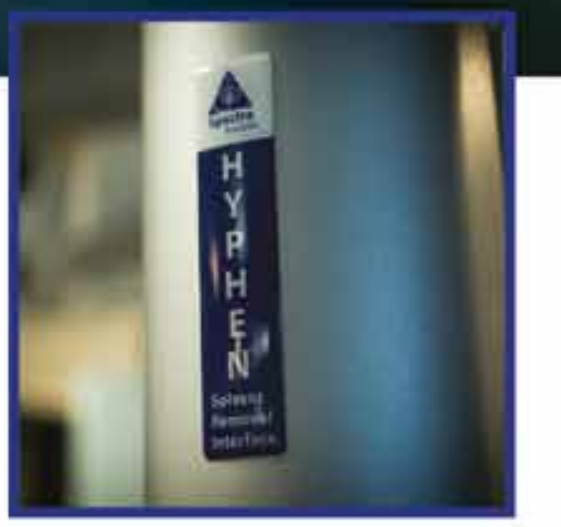

\section{ПРЕИМУЩЕСТВА}

- Конфигурация DiscovIR-GC для работы с любыми газовыми хроматографами.

- Конфигурация DiscovIR-LC для систем гельпроникающей, эксклюзионной и высокоэффективной жидкостной хроматографии.

- Соединение ВЭЖX с системой DiscovIR-LC с использованием уникального интерфейса с полным удалением растворителя.

- осаждение микроколичеств образца на выходе из колонки на охлаждаемый жидким азотом диск.

- Анализ твердых образцов с высоким разрешением.

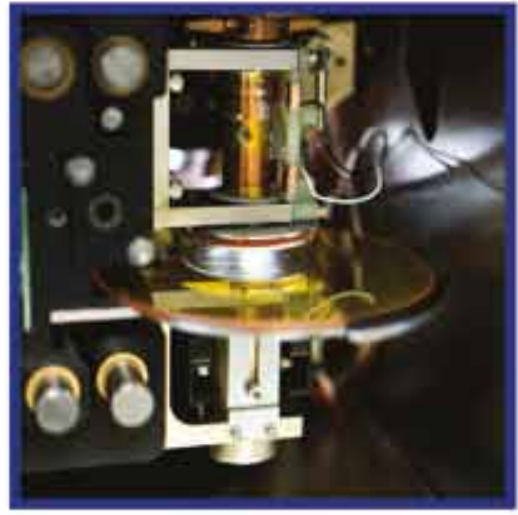

- Библиотеки спектров включают более чем 2500 соединений.

\section{ХАРАКТЕРИСТИКИ}

- Диапазон ИК: 4000-700 cм'.

- Разрешение: 4 или $8 \mathrm{~cm}^{-1}$.

- ИК-детектор: 0,1 мм × 0,1 мм КРТ-приемник.

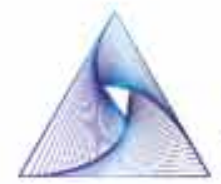

